\title{
A Study on Efficacy and Safety of Silodosin in Geriatric Patients with Benign Prostatic Hyperplasia
}

\author{
Dr. Chilukala Sai Praneeth ${ }^{1}$, Dr. Rajeev T.P ${ }^{2}$, Dr. Shubhakar Bhandary ${ }^{1}$, \\ Dr. Chaitanya Reddy \\ 1.Department of General Surgery, 2. Department of Urology, K.S. Hegde Medical Academy, \\ Deralakatte, Mangalore - 575018, Karnataka, India
}

\begin{abstract}
Introduction: Benign prostatic hyperplasia $(B P H)$ is a very common condition among elderly men causing significant symptoms with urination and results in bladder outlet obstruction. Silodosin is a recently developed selective alA-adrenoreceptor antagonist for the medical treatment of BPH. This study is to evaluate the efficacy and safety of silodosin $8 \mathrm{mg}$ in geriatric patients with $\mathrm{BPH}$.

Materials And Methods: A prospective observational study was done at K.S.Hegde Medical Academy, Mangalore, India on 53 patients with BPH during August 2014 to march 2016. These patients were aged $\geq 50$ years, with international prostate symptom score(IPSS) of $\geq 12$ and having a peak urinary flow rate $(P F R)<15$ $\mathrm{ml} / \mathrm{sec}$ on uroflowmetry. Patients were treated with silodosin $8 \mathrm{mg}$ once daily and were followed up. We assessed the changes in IPSS,PFR and adverse effects of the drug at baseline, 1 month and 3 months after treatment.

Results: The mean age of subjects was 63.32 \pm 6.6 . Their mean IPSS scores were 25.06 $\pm 2.87,18.91 \pm 3.92$, $16.19 \pm 4.9$ at baseline, 1month and 3 months respectively with significant improvement $(p<0.0001)$. There was also a significant increase in the peak urinary flow rate $(P F R)(p<0.0001)$. The only adverse effect encountered was retrograde ejaculation. In three out of 5 patients this problem subsided without any intervention.

Conclusions: Silodosin is an effective and well tolerated drug producing significant improvement in IPSS and PFR with fewer cardiovascular side effects in this frail population. Therefore silodosin can be used as a first line agent in BPH and continuation of this drug will avoid a surgical intervention.
\end{abstract}

Keywords: Silodosin, Benign Prostatic Hyperplasia, IPSS, Peak flow rate, obstructive symptoms, storage symptoms.

\section{Introduction}

$\mathrm{BPH}$ is a common clinical entity in geriatric age group, occurring in upto $70 \%$ of men over the age of 60 years, and in more than $90 \%$ of those aged over 80 years [1]. Histologically, BPH is a non-malignant enlargement of the prostate characterised by cellular hyperplasia of both glandular and stromal elements[2]. Clinically it presents as lower urinary tract symptoms (LUTS: increased frequency, urgency, a weak intermittent urinary stream, strain during micturition, a sense of incomplete emptying and nocturia), which further leads to complications, like acute urinary retention [3]. However the severity of lower urinary tract symptoms(LUTS) is not related to the prostate size or Bladder Outlet Obstruction(BOO) [4,5]. The severityof LUTS seems to be linked with, to some extent, smooth muscle tone in the prostate and bladder neck [5]. The treatment options for BPH include alpha-blockers, 5 $\alpha$-reductase inhibitors, Transurethral Resection of the Prostate (TURP), transurethral ablative modalities, open prostatectomy and herbal treatments (saw palmetto plant extracts). According to the guidelines of EAU update March 2015, $\alpha$-blockers are the recommended first-line therapy for all men with moderate to severe LUTS [6].

Studies have shown that there are numerous $\alpha 1 \mathrm{~A}$ - Adrenoreceptors in the prostate and the bladder neck which mediate the smooth muscle. On the other hand, $\alpha 1 \mathrm{~B}$-ARs are largely located on vascular smooth muscle which regulate the blood pressure and the contraction of peripheral vasculature $[7,8]$. Therefore, the drugs which are $\alpha 1 \mathrm{~A}$-Adrenoreceptor selective are beneficial in treating BPH with fewer cardiovascular effects as seen in non selective $\alpha$-blockers. Silodosin is an emerging agent with high selectivity for $\alpha 1 \mathrm{~A}$-ARs recently developed in Japan. In vitro studies have showed that silodosin's $\alpha 1 \mathrm{~A}$-to- $\alpha 1 \mathrm{~B}$ binding ratio is extremely high (162:1) [9]. Schwinn DA et al [10], Forray C et al [11] compared silodosin with non-selective alpha1A-adrenoceptor (AR) blockers and demonstrated that drugs with a high selectivity for $\alpha 1 \mathrm{~A}$-Adrenoreceptors may be more prostatespecific and maintain a therapeutic response in the treatment of symptomatic BPH with minimal effect on blood pressure and fewer cardiovascular side effects. With this above mentioned background, a prospective observational study was done on the efficacy and safety of silodosin $8 \mathrm{mg}$ once daily in geriatric patients suffering from BPH for a period of 3 months. 


\section{Materials Aend Methods}

Study Design: An observational, prospective study was conducted on 53cases diagnosed with BPH in the Urology department of K.S Hegde Medical Academy, Mangalore, South India during a period of 12 months from August 2014 to July 2015 . Patients presented with prostate related symptoms and diagnosis was confirmed both clinically and sonologically. Serum prostate specific antigen (PSA) was estimated to rule out malignancy. The ethical committee of the institution approved this clinical study and each subject gave written informed consent before beginning any investigational procedure. A total of 3 visits were foreseen from each patient i.e. at baseline, 1 month and 3 months after the initiation of therapy. Data was collected regarding clinical history (including IPSS and adverse effects), manual blood pressure and Uroflowmetry analysis(PFR) in each visit. The inclusion criteria for this study was kept as age greater than or equal to 50 years, Peak urinary flow rate at least $4 \mathrm{ml} / \mathrm{sec}$ but not greater than $15 \mathrm{ml} / \mathrm{sec}$ and a voided volume of atleast $150 \mathrm{ml}$ on uroflowmetry and an International Prostate Symptom Score(IPSS) of 12 or higher associated with moderate to severe symptoms. Patients with supine blood pressure less than $90 / 70 \mathrm{~mm}$ of $\mathrm{Hg}$, patients with a history of prior surgical intervention for BPH and patients with neurogenic bladder, prostate carcinoma, stricture urethra, bladder stone, recent gross haematuria, prostatic abscess were excluded from the study.

\section{Objectives of the study:}

1) To assess the change in peak urinary flow rate.

2) To assess the changes in the IPSS.

3) To observe for side effects of the therapy

The primary endpoint of this study was to measure the changes in IPSS total score, peak urinary flow rate(PFR) and adverse effects of therapy in all 3 visits(primary efficacy variables). The secondary efficacy variables evaluated in this study were changes in IPSS obstructive and storage symptom subscores.

\section{Statistical Analysis}

Total 53 geriatric patients diagnosed to have BPH either by clinical evaluation or ultrasonographically are taken into this study and they were started on $8 \mathrm{mg}$ of silodosin once daily therapy for 3 months. The mean age of subjects in our study was $63.32 \pm 6.6$. Data related to international prostate symptom score(IPSS), obstructive symptoms subscore, irritative/storage symptoms subscore, peak urinary flow rate(PFR) and adverse effects of the drug had been assessed before treatment, 1month and 3 months after treatment.

The collected information was summarized by using mean \pm standard deviation (S.D). The difference between IPSS, obstructive, storage symptom scores and PFR from baseline to 1 month and 3 months was analysed by using repeated measures ANOVA with Bonferroni adjustment (multiple comparisons). Their mean IPSS scores were $25.06 \pm 2.87,18.91 \pm 3.92,16.19 \pm 4.9$ at baseline, 1 month and 3 months after initiation of treatment respectively and there was a significant reduction in the IPSS scores from baseline to 1 month $(\mathrm{p}<0.0001)$ and baseline to 3 months $(\mathrm{p}<0.0001)$.

\section{Descriptive Statistics}




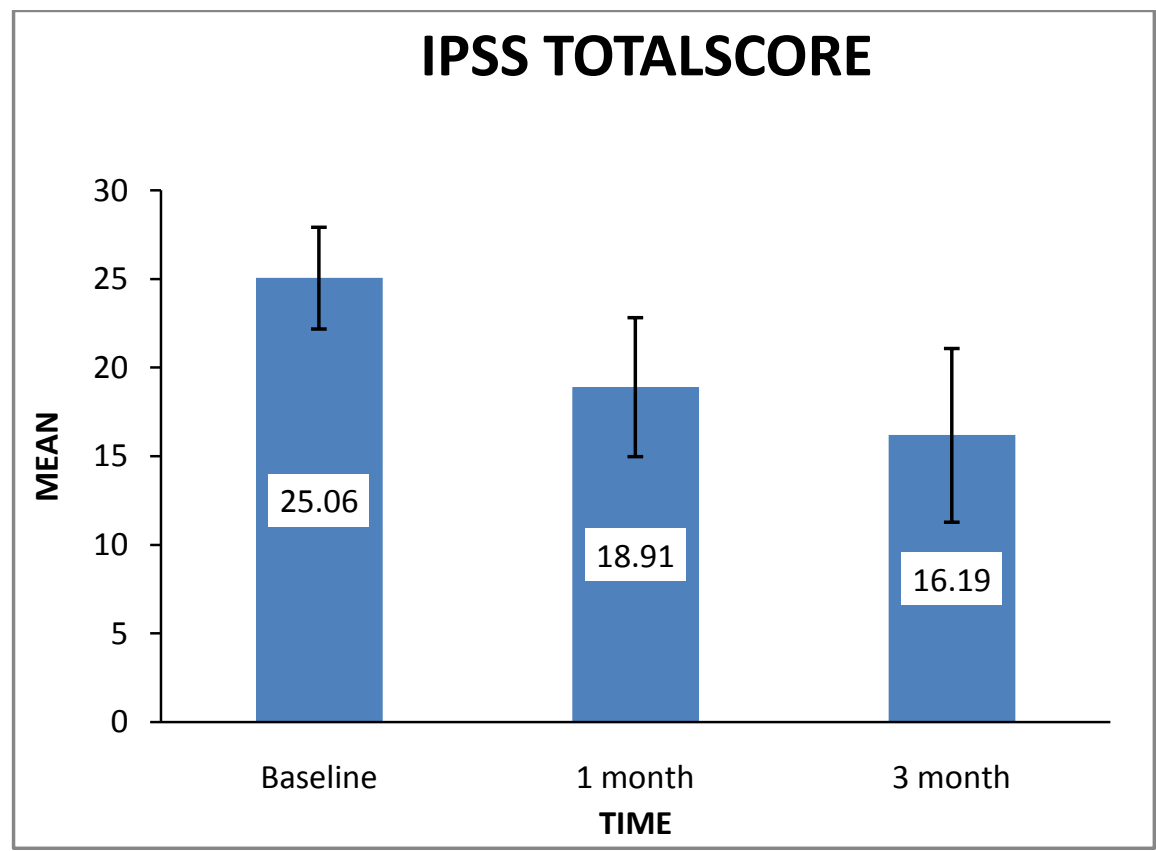

Chart:1

The obstructive symptoms include poor stream, intermittency, straining and sensation of incomplete micturition. The mean subcoring of obstructive symptoms were $15.2 \pm 2.07,11.26 \pm 2.1$ and $9.85 \pm 3.14$ at baseline, 1 month and 3 months after initiation of treatment respectively. There was a significant reduction in the obstructive symptom subscores from baseline to 1 month $(\mathrm{p}<0.0001)$ and baseline to 3 months $(\mathrm{p}<0.0001)$.

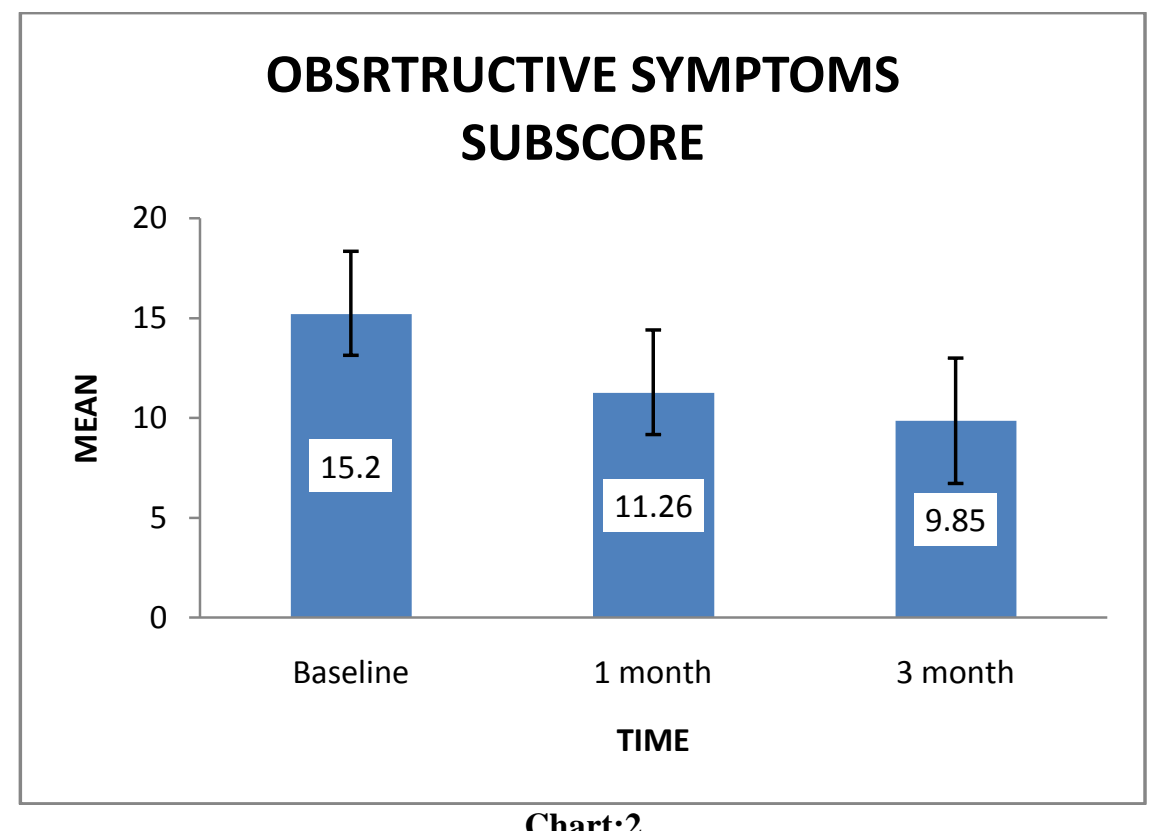

\section{Chart:2}

The irritative or storage symptoms include frequency, urgency and nocturia. The mean storage symptom subscorings were $10.21 \pm 3.09,7.94 \pm 3.26,6.81 \pm 3.17$ at baseline, 1 month and 3 months after initiation of treatment respectively and there was a significant reduction in the IPSS scores from baseline to 1 month $(\mathrm{p}<0.0001)$ and baseline to 3 months $(\mathrm{p}<0.0001)$. 


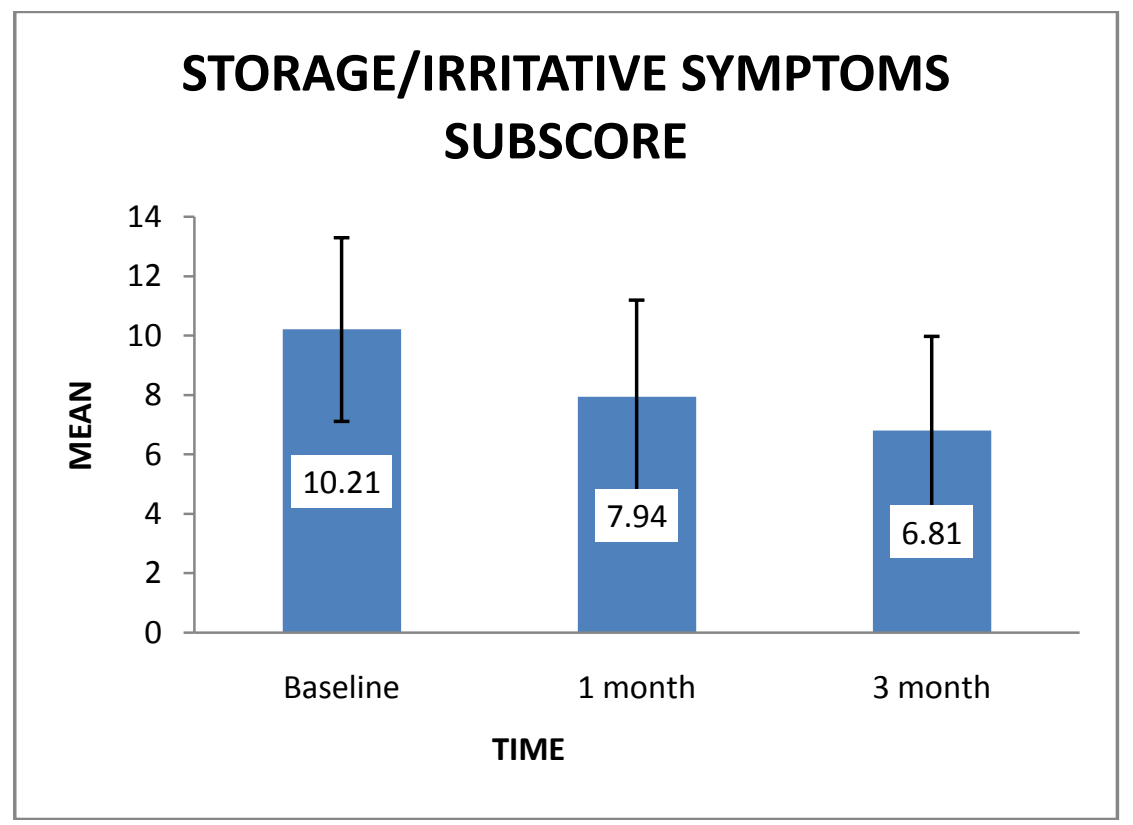

\section{Chart:3}

There was also a significant increase in the peak urinary flow rate (PFR) $(\mathrm{p}<0.0001)$ with mean PFR values $9.29 \pm 2.41,11.6 \pm 2.34$ and $13.01 \pm 2.96$ at baseline, 1 month and 3 months respectively.

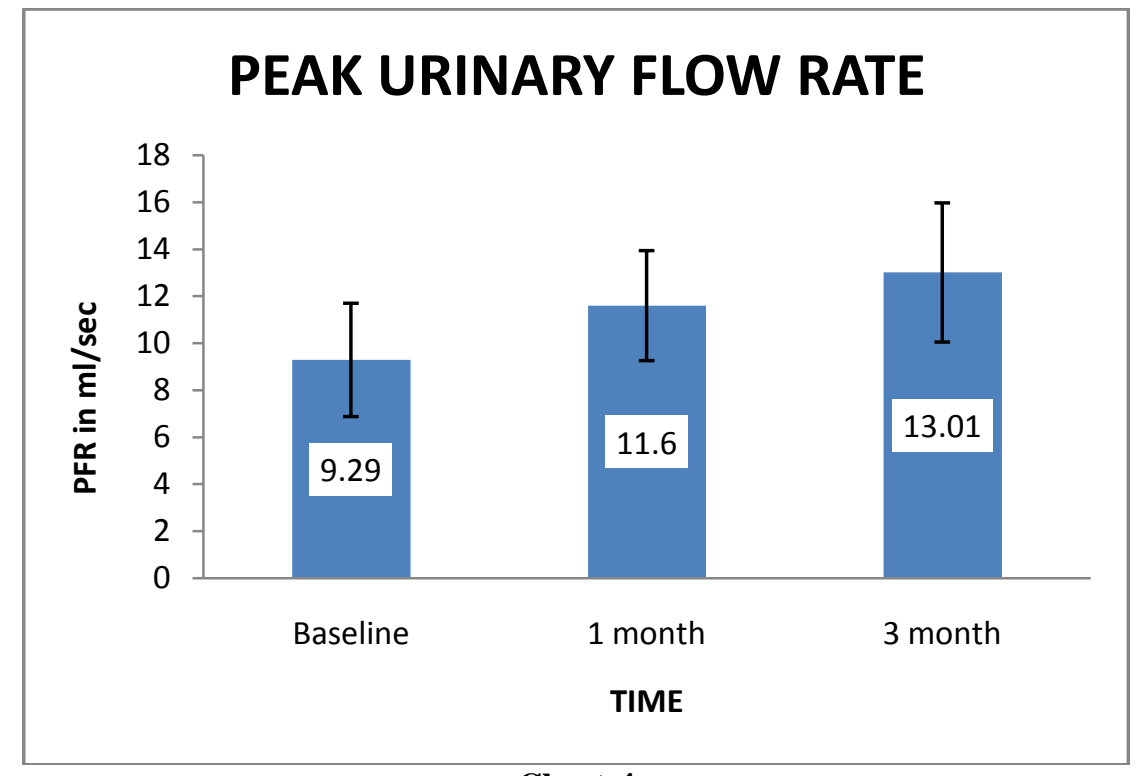

\section{Chart:4}

The only adverse effect encountered with silodosin was a bothersome abnormal ejaculation in 5 patients within 1 month of treatment. In three out of 5 patients this problem subsided within 3 months without any intervention. None of the patients had progression of disease or complications related to BPH requiring surgical intervention during the study period.

\section{Discussion}

The medical management of BPH has several advantages over surgical procedures in relation to its effectiveness, safety, and reduced number of associated side effects. The goal of medical management of BPH is to alleviate symptoms, to relieve smooth muscle contractions of the prostatic capsule and bladder neck and urination resistance, and to prevent urine retention, urinary tract infection and renal failure. Current pharmacological therapies for this condition can be classified into six major categories: (1) $\alpha 1$-adrenoceptor blockers, (2) $5 \alpha$-reductase inhibitors, (3) muscuranic receptor antagonists, (4) phosphodiesterase inhibitors, (5) 
vasopressin analogues, (6) combination therapies. There are four subtypes of $\alpha 1$-adrenoceptors, of which the $\alpha 1 \mathrm{~A}$-adrenoceptor is typically located in the prostate, accounting for $70 \%$ of $\alpha 1$-adrenoceptors in the prostate $[10,12]$. Silodosin is a new selective $\alpha 1$ A-antagonist that has been approved by the US Food and Drug Administration for the treatment of BPH since October 2008 [14].

Alpha1-ARs are a family of $\mathrm{G}$ protein-coupled receptors. The bindingof norepinephrine and epinephrine induces phospholipase $\mathrm{C}$ activation, leading to the generation of secondary messengers, including inositol triphosphate and diacylglycerol. Finally, this binding induces an increase in the intracellular calcium levels and smooth muscle contraction [15]. The main cause of LUTS in BPH is prostate contraction that is predominantly mediated by alpha1A-AR[16,17]. Therefore, the blockage of $\alpha 1 \mathrm{~A}$ adrenoreceptors results in prostatic and urethral smooth muscle relaxation and may improve the voiding symptoms. Yokoyama et al[18]demonstrated that the selective $\alpha 1 \mathrm{~A}$-blocker silodosin exerts inhibitory effects on detrusor overactivity by modulating $\mathrm{C}$-fibre afferent activity using animal models. Consequently, $\alpha 1 \mathrm{~A}$-blockade can improve the storage symptoms.

Moon et al [19] in Korea have conducted a prospective, single arm study on efficacy and safety of silodosin for lower urinary tract symptoms associated with BPH in 2011 on 100 subjects and their studies showed a significant improvement in IPSS scores $(23.27+3.34,15.89+6.26$ and $13.80+6.31$ at baseline, 4weeks and 12 weeks respectively) and differences were statistically significant $(\mathrm{p}<0.0001)$. There was significant difference in PFR between baseline and 12 weeks $(p<0.0001)$ but not in PVR $(p=0.9404)$. During the study the most frequent adverse effect was ejaculation failure in 13 cases. However, no subject dropped out because of ejaculation failure and in 12 out of 13 cases it was fully resolved without further treatment.

Kawabe et al [20] investigated the efficacy and safety of long-term administration of silodosin in patients with LUTS associated with BPH. The results showed that statistically significant improvement in the total IPSS, in IPSS subscores, in Qmax and QoL all sustained for 52 weeks from as early as week 4.

However Chapple et al [21] reported that the frequency of ejaculation disorder was $14.2 \%$ in the silodosin treatment group, which was also significantly higher compared with 2.1 in the $0.4 \mathrm{mg}$ tamsulosin treatment group. Song et al. [22] reported that the overall incidence of ejaculatory dysfunction was $13.4 \%$ after 12 weeks of $0.2 \mathrm{mg}$ tamsulosin treatment. Retrograde ejaculation is caused by smooth muscle relaxation in the prostate, urethra, bladder neck and vas deferens. The $\alpha 1 \mathrm{~A}$-adrenoreceptor is mainly expressed in the bladder neck, vas deferens and seminal vesicles. Moreover, Moriyama et al [23] showed that the $\alpha 1 \mathrm{~A}$-adrenoreceptor subtype mediates human vas deferens contraction. Therefore, this adverse effect is explained by the high $\alpha 1 \mathrm{~A}-$ adrenoreceptor subtype selectivity of silodosin.

Homma et al [24] performed a post hoc analysis of the data from a randomized, double-blind, placebocontrolled clinical trial of silodosin in Japan and found that ejaculation disorder caused by selective $\alpha 1 \mathrm{~A}$ blockers was associated with very large improvements in the lower urinary tract symptoms. The silodosin subgroup with ejaculation failure experienced a greater reduction in the total IPSS than the silodosin subgroup without ejaculation impairmentand the placebo subgroup ( 211.8 vs. 27.2 vs. 25.3 , respectively). However, there was no difference in the discontinuation rates between the silodosin subgroup with ejaculation disorder and the silodosin subgroup without ejaculation disorder. Thus, ejaculation disorder may be a predictor of the efficacy of the $\alpha 1 \mathrm{~A}$-adrenoreceptor blockade.

A recent RCT [21] suggested that compared with tamsulosin or placebo, silodosin showed no significant differences in the supine systolic blood pressure, diastolic blood pressure and heart rate. $\alpha 1 \mathrm{~B}$ adrenoreceptors have been demonstrated to mediate both blood vessel contraction and baroreceptor-induced inotropic effects [25,26,27]. Moreover, tamsulosin has a higher selectivity for the $\alpha 1 \mathrm{~B}$-adrenoreceptors than silodosin, and thus, the decrease in blood pressure induced by tamsulosin may be mediated by its blocking $\alpha 1 \mathrm{~B}-$ adrenoreceptors action on the participating in blood vessel contraction and baroreceptor-induced inotropic effects.[9,16] Therefore, the lack of cardiovascular side effects may be a major advantage of silodosin. Our study statistically proved that silodosin is effective in improving the IPSS, PFR with fewer cardiovascular side effects. None of the patients required a surgical intervention during study period.

\section{Conclusions}

Silodosin is an effective and well tolerated drug in geriatric patients suffering from benign prostatic hyperplasia (BPH). Despite abnormal ejaculation, the overall efficacy of silodosin in alleviating the symptoms both obstructive and storage, and improving the peak urinary flow rate is proven. It carries a high degree of safety in this frail population compared to other $\alpha$ blockers with respect to cardiovascular side effects. Therefore silodosin can be used as a first line agent in the medical management of BPH and continuation of this drug will avoid surgical intervention in geriatric population.

\section{References}

[1]. Berry SJ, Coffey DS, Walsh PC, Edwin LL. The development of human benign prostatic hyperplasia with age. J Urol 1984; 132: 474-479. 
[2]. Chapple C. Medical treatment for benign prostatic hyperplasia. BMJ $\quad$ 1992; 304:1198-1199

[3]. McNicholasT,Kirby R. Benign prostatic hyperplasia and male lower urinary tract symptoms (LUTS). ClinEvid (Online) 2011; pii: 1801.

[4]. Barry MJ, Cockett AT, Holtgrewe HL, McConnell JD, Sihelnik SA, Winfield HN.Relationship of symptoms of prostatism to commonly used physiological and anatomical measures of the severity of benign prostatic hyperplasia. J Urol 1993;150: 351358.

[5]. Andersson KE, Gratzke C. Pharmacology of a1-adrenoceptor antagonists in the lower urinary tract and central nervous system. Nat ClinPractUrol 2007; 4: 368-378.

[6]. S. Gravas (Chair), T. Bach, A. Bachmann, M. Drake, M. Gacci, C. Gratzke, S. Madersbacher, C. Mamoulakis, K.A.O. Tikkinen management of non - neurogenic male lower urinary tract symptoms (LUTS) - update March 2015 p15

[7]. Roehrborn CG, Schwinn DA. $\alpha 1$-Adrenergic receptors and their inhibitors in lower urinary tract symptoms and benign prostatic hyperplasia. J Urol 2004; 171: 1029-1035.

[8]. Kirby R, Andersson KE, Lepor H, Steers WD. $\alpha 1$-Adrenoceptor selectivity and the treatment of benign prostatic hyperplasia and lower urinary tract symptoms. Prostate Cancer Prostatic Dis 2000; 3: 76-83.

[9]. Tatemichi S, Kobayashi K, Maezawa A, Kobayashi M,Yamazaki Y, Shibata N. a1-Adenoceptor subtype selectivity and organ specificity of silodosin(KMD-3213). YakugakuZasshi 2006; 126: 209-216.

[10]. Schwinn DA, Roehrborn CG. Alpha1-adrenoceptor subtypes and lower urinary tract symptoms. Int J Urol 2008; 15 : 193-9.

[11]. Forray C, Bard JA, Wetzel JM, Chiu G, Shapiro E et al. The alpha1-adrenergic receptor that mediates smooth muscle contraction in human prostate has the pharmacological properties of the cloned human alpha 1c subtype. MolPharmacol 1994; 45: 703-8.

[12]. Shibata K, Foglar R, Horie K et al (1995) KMD-3213, a novel, potent; a1 A-adenoceptor-selective antagonist characterization using recombinant human a1-adenoceptors and native tissues. MolPharmacol 48:250-258.

[13]. Schilit S, Benzeroual KE. Silodosin: a selective alpha1A-adrenergic receptor antagonist for the treatment of benign prostatic hyperplasia. ClinTher 2009; 31: 2489-502.

[14]. Rossi M, Roumeguere T. Silodosin in the treatment of benign prostatic hyperplasia.Drug Des DevelTher 2010; 4: $291-7$.

[15]. Michel MC, Vrydag W. Alpha1-, alpha2- and beta-adrenoceptors in the urinary bladder, urethra and prostate. Br J Pharmacol 2006; 147(Suppl 2)S88-119.

[16]. Roehrborn CG. Efficacy of alpha-adrenergic receptor blockers in the treatment of male lower urinary tract symptoms. Rev Urol 2009; 11: S1-8.

[17]. Yokoyama O, Ito H, Aoki Y, Oyama N, Miwa Y et al. Selective alpha1A-blocker improves bladder storage function in rats via suppression of C-fiber afferent activity. World J Urol 2010; 28: 609-14.

[18]. Efficacy and safety of the selective $\alpha 1 \mathrm{~A}$ adrenoreceptor blocker silodosin for severe lower urinary tract symptoms associated with BPH: a prospective, single open label, multicenter study in Korea.Korean Journal of Urology 2014;55:335-340 doi:10.4111/kju.2014.55.5.335

[19]. Kawabe K, Yoshida M, Arakawa S, Takeuchi H. Long-term evaluation of silodosin, a new a1A-adrenoceptor selective antagonist for the treatment of benign prostatic hyperplasia: Phase III long-term study. Jap J UrolSurg 2006; 19:153-164.

[20]. Chapple CR, Montorsi F, Tammela TL, Wirth M, Koldewijn E et al. Silodosin therapy for lower urinary tract symptoms in men with suspected benign prostatic hyperplasia: results of an international, randomized, double-blind, placebo- and activecontrolled clinical trial performed in Europe. EurUrol 2011; 59: 342-52.

[21]. Song SH, Son H, Kim KT, Kim SW, Moon duG et al. Effect of tamsulosin on ejaculatory function in BPH/LUTS. Asian J Androl 2011; 13: 846-50.

[22]. Moriyama N, Nasu K, Takeuchi T, Akiyama K, Murata S et al. Quantification and distribution of alpha 1-adrenoceptor subtype mRNAs in human vas deferens: comparison with those of epididymal and pelvic portions. Br J Pharmacol 1997;122: 1009-14

[23]. Homma Y, Kawabe K, Takeda M, Yoshida M. Ejaculation disorder is associated with increased efficacy of silodosin for benign prostatic hyperplasia. Urology 2010; 76:1446-50

[24]. Hatano A, Takahashi H, Tamaki M, Komeyama T, Koizumi T et al. Pharmacologicalevidence of distinct alpha 1-adrenoceptor subtypes mediating the contraction ofhuman prostatic urethra and peripheral artery. Br J Pharmacol 1994; 113: 723-8.

[25]. Take H, Shibata K, Awaji T, Hirasawa A, Ikegaki I et al. Vascular alpha1-adrenoceptorsubtype selectivity and alpha1-blockerinduced orthostatic hypotension.JpnJPharmacol 1998; 77: 61-70.

[26]. Townsend SA, Jung AS, Hoe YS, Lefkowitz RY, Khan SA et al. Critical role for thealpha-1B adrenergic receptor at the sympathetic neuroeffector junction.Hypertension 2004; 44: 776-82.

Chart: 1 - changes in IPSS score

\section{Legends}

Chart:2 - changes in Obstructive symptom subscores

Chart:3 - changes in storage symptom subscores

Chart:4 - changes in PFR. 